\title{
Dexmedetomidine or midazolam in combination with propofol for sedation in endoscopic retrograde cholangiopancreatography: a randomized double blind prospective study
}

\author{
Senem Koruk ${ }^{1}$, Irfan Koruk ${ }^{2}$, Ayse Mizrak Arslan ${ }^{3}$, Murat Bilgi $^{4}$, Rauf Gul ${ }^{5}$, Semsettin Bozgeyik ${ }^{6}$ \\ ${ }^{1}$ Department of Anesthesiology and Reanimation, Faculty of Medicine, Istanbul Medeniyet University, Istanbul, Turkey \\ ${ }^{2}$ Department of Gastroenterology, Faculty of Medicine, Demiroglu Bilim University, Istanbul, Turkey \\ ${ }^{3}$ Department of Anesthesiology and Reanimation, Gaziantep University Faculty of Medicine, Gaziantep, Turkey \\ ${ }^{4}$ Department of Anesthesiology and Reanimation, Abant Izzet Baysal University, Bolu, Turkey \\ ${ }^{5}$ Department of Anesthesiology and Reanimation, NCR International Hospital, Gaziantep, Turkey \\ ${ }^{6}$ Department of Anesthesiology and Reanimation, Defa Life Hospital, Gaziantep, Turkey
}

Videosurgery Miniinv 2020; 15 (3): 526-532 DOI: https://doi.org/10.5114/wiitm.2020.95066

\begin{abstract}
Introduction: Interventional endoscopic procedures, such as endoscopic retrograde cholangiopancreatography $(E R C P)$, often require sedation during the procedure. The most commonly used drugs for this purpose are midazolam and propofol, which are used as sedative and hypnotic agents with minimal analgesic potential.

Aim: To compare the analgesic sedative effects of midazolam-propofol and dexmedetomidine-propofol combinations and their influence on hemodynamic and respiratory variables in patients undergoing ERCP.

Material and methods: Forty adult patients aged 20-78 and undergoing ERCP were randomized to two groups. Patients were premedicated with midazolam $(0.05 \mathrm{mg} / \mathrm{kg} 10 \mathrm{~min}$ before the procedure) in group $M$ and with dexmedetomidine (1 $\mathrm{\mu g} / \mathrm{kg}$ for $10 \mathrm{~min}$ ) in group D. Propofol was used for maintenance. The sedation level was monitored using the bispectral index (BIS) to maintain a score between 70 and 80. Hemodynamic and respiratory variables, recovery time and adverse events were recorded.

Results: The hemodynamic and respiratory variables were similar in both groups. Total propofol consumption was significantly lower in the dexmedetomidine group (208.5 $\pm 80.0 \mathrm{vs}$. $154.5 \pm 66.7 \mathrm{mg} ; p=0.011)$. The recovery period was shorter in group D (time to achieve the Aldrete score 9 was $9.4 \pm 2.1$ vs. $6.6 \pm 1.1 \mathrm{~min} ; p<0.001$ ). Changes in hemodynamic and respiratory variables and adverse events were not different between the two groups.

Conclusions: We found a shorter recovery time and comparable sedative and adverse effects with the dexmedetomidine-propofol combination compared with the midazolam-propofol combination. Dexmedetomidine in combination with propofol may be a safe and useful alternative for sedation for ERCP patients.
\end{abstract}

Key words: dexmedetomidine, sedation, bispectral index, endoscopic retrograde cholangiopancreatography, recovery time.

\section{Introduction}

Interventional endoscopic procedures, such as endoscopic retrograde cholangiopancreatography (ERCP), often require sedation. The most commonly used agent for this purpose is midazolam. This drug has a short elimination half-life and has amnestic and anxiolytic effects [1]. Propofol is a widely used sedative 
and hypnotic agent with minimal analgesic potential. Propofol may cause respiratory depression, especially in high doses [2]. To reduce cumulative propofol doses, it may be used in combination with other drugs, such as midazolam or dexmedetomidine. Propofol and midazolam act synergistically in combination and may be more effective than when used alone [3]. Dexmedetomidine is a highly selective $\alpha 2$-adrenergic receptor agonist that has analgesic and sedative effects with minimal depression on ventilation [4]. It has been reported that dexmedetomidine reduces the recruitment of propofol during anaesthesia because dexmedetomidine has analgesic effects [5].

The level of sedation can easily shift from conscious to deep sedation and result in the loss of protective reflexes and may cause problems in airway control [3]. Therefore, the sedation level should be monitored and managed carefully. For assessment of the level of sedation, bispectral index (BIS) monitoring may be used; it is an objective method and provides titration of drugs $[6,7]$. The BIS is a complex mathematical evaluation of relevant, descriptive electroencephalographic parameters of the frontal cortex corresponding to varying levels of sedation [8]. Patients undergoing general anaesthesia require a BIS level of 40 to 60 , and a level of approximately 80 is adequate for less invasive procedures, such as ERCP and endoscopic interventions [9].

\section{Aim}

In this study, the primary aim was to compare the recovery time after propofol consumption between the dexmedetomidine-propofol group and midazolam-propofol group in patients undergoing ERCP. The secondary outcome was to compare cardiorespiratory responses of the groups. We hypothesized that a combination of dexmedetomidine-propofol would provide a shorter recovery time than a midazolam-propofol combination without respiratory side effect and haemodynamic instability.

\section{Material and methods}

This randomized, prospective and double-blind study was conducted with the approval of the university ethics committee. This study is registered and approved by the University Medical Ethics Committee with the registration number of 04-2009/112, 09.04.2009. We obtained written informed consent from all patients. Forty patients scheduled for ther- apeutic ERCP aged between 20 and 78 years were enrolled in the study. All patients were in ASA I-III physical status. Patients under the age of 18, those who were pregnant, chronically using opioid or $\alpha 2$ agonist drugs, had a history of allergy to one of the drugs used in the study, had severe cardiac or respiratory comorbidity, had second- or third-degree heart block, had ASA IV-V status, had a body mass index (BMI) over $36 \mathrm{~kg} / \mathrm{m}^{2}$ and those who refused to participate in the study were excluded from the study. The patients were randomized to two groups using a computer by a physician who did not follow up the patients during the procedure (Figure 1). After being taken to the operating room, patients were monitored for heart rate (HR), mean arterial blood pressure (MAP), respiratory rate (RR) and arterial oxygen saturation $\left(\mathrm{SpO}_{2}\right)$, and the baseline measurements of haemodynamic variables were recorded. BIS monitoring (Aspect Medical Systems, Natick, Mass, US) was also applied to all patients. BIS values range between 0 and 100 (0: no cortical activity or coma; 40-60: unconscious; 70-90: varying levels of conscious sedation; 100: fully awake). In this study, the Bis value was maintained at 70-80, which was sufficient for conscious sedation. After recording the basal values, we used a topical anaesthetic (Vemcaine pump spray $10 \%$, VEM Pharmaceuticals Industry and Trade Co., Tekirdag, Turkey) for pharyngeal anaesthesia. Patients were premedicated by a physician who was informed about the randomization. In one group (group $M ; n=20$ ), patients were premedicated with midazolam $0.05 \mathrm{mg} / \mathrm{kg}$ (IV) $10 \mathrm{~min}$ before

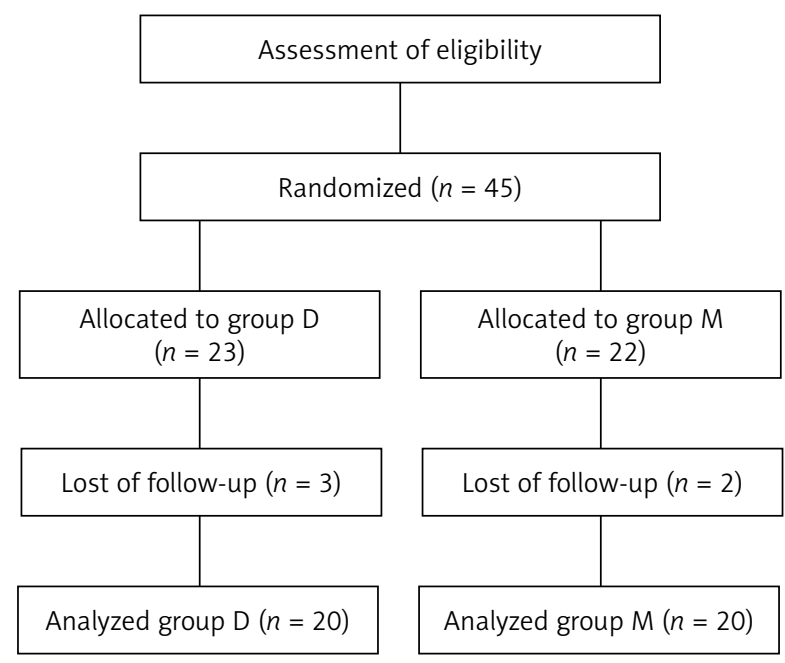

Figure 1. Consort diagram 
propofol administration. In the other group (group D; $n=20$ ), patients received dexmedetomidine (Precedex $200 \mu \mathrm{g}$, Hospira, Rocky Mount, USA) $1 \mu \mathrm{g} / \mathrm{kg}$ (IV injected in $10 \mathrm{~min}$ ) prior to the propofol administration. The physician who applied the premedication did not participate in the patient's follow-up to maintain study blindness. Two other physicians followed the patients after premedication. Propofol (propofol 1\% Fresenius Kabi USA, LLC, Lake Zurich) in the same doses $(1-1.5 \mathrm{mg} / \mathrm{kg}$ for first bolus dose and intermittent doses of $20 \mathrm{mg}$ to achieve a BIS score between 70 and 80) was used for sedation in both groups. ERCP was initiated after achieving an adequate sedation level (BIS: 70-80). The ERCP was performed by an experienced gastroenterologist (over 3000 therapeutic ERCP procedures) in a standard manner. The data ( $\mathrm{HR}, \mathrm{MAP}, \mathrm{RR}$ and $\mathrm{SpO}_{2}$ ) were recorded at 5-min intervals during the procedure. When there was more than a $20 \%$ increase or decrease in heart rate and blood pressure, it was evaluated as a side effect. Other side effects (such as arrhythmias, nausea-vomiting and shivering) were also recorded. In the case of bradycardia ( $\mathrm{HR}<40$ beat/ min), hypotension (MAP $<50 \mathrm{~mm} \mathrm{Hg}$ ), bradypnea (RR $<10 / \mathrm{min})$ or desaturation $\left(\mathrm{SpO}_{2}<92 \%\right)$, adequate therapeutic applications were carried out in each situation (atropine $0.5 \mathrm{mg}$ for bradycardia, 0.9\% saline infusion $(500 \mathrm{ml} / \mathrm{h})$ for hypotension, and oxygen supply (4 l/min) with a nasal cannula for desaturation were the planned treatments). In the event of respiratory depression, patients were planned to be supported with a jaw thrust maneuver and ventilation with a balloon mask. During the procedure, propofol doses were also administered to maintain BIS levels between 70 and 80, and the cumulative dose was recorded. With termination of ERCP, drugs were ceased, and patients were evaluated with an
Aldrete score [10] for defining the recovery period by physicians blinded to the drug regimen. The period between the termination of ERCP and time to achieve an Aldrete score of 9 was accepted as the recovery time.

\section{Statistical analysis}

The data were analysed using the IBM SPSS Statistics for Windows, version 22 (IBM Corp., Armonk, N.Y., USA) program. All data were analysed in terms of mean \pm standard deviation (SD). After the preliminary study with 10 patients, we performed a power analysis by using the recovery time. We calculated the sample size as 26 in total ( $\alpha=0.05$; power $=$ 0.95). We performed this study with 40 patients, and post hoc power analysis (G Power 3.1.9.2) according to recovery time with 40 patients was performed; for $\alpha=0.05$ and power $=0.99$, the effect size was 1.897 . For continuous variables, Student's $t$ test was used to compare differences between groups. Category variables were analysed with the $\chi^{2}$ or Fisher's exact test as appropriate. For all tests, a $p$-value $<0.05$ was considered to be significant.

\section{Results}

Forty patients were enrolled in the study. The demographic parameters of patients were similar between groups. The age, gender, height and body weight, ASA physical status and operation time are shown in Table I. Changes in blood pressure and heart rate were similar in both groups (Figures 2 and 3). When the groups were compared according to the recovery time with the Aldrete score, the recovery time to achieve the Aldrete score 9 was found to be shorter in group $D$ than in group $M(6.6 \pm 1.1 \mathrm{~min}$ in group $D$ vs. $9.4 \pm 2.1 \mathrm{~min}$ in group $M$, respectively;

Table I. Demographic variables of patients in groups

\begin{tabular}{|lccc|}
\hline Parameter & Group M $(n=20)$ & Group D $(n=20)$ & $P$-value \\
\hline Age [year] & $50.8 \pm 16.1$ & $53.3 \pm 11.0$ & NS \\
\hline Gender M/F & $9 / 11$ & $9 / 11$ & NS \\
\hline Height [m] & $1.66 \pm 0.06$ & $1.67 \pm 0.06$ & NS \\
\hline Body weight [kg] & $71.0 \pm 9.31$ & $69.4 \pm 12.0$ & NS \\
\hline ASA I/II/III & $3 / 16 / 1$ & $2 / 17 / 1$ & NS \\
\hline Procedure time [min] & $16.7 \pm 4.2$ & $18.8 \pm 5.5$ & \\
\hline
\end{tabular}

$P<0.05$ is considered statistically significant. 


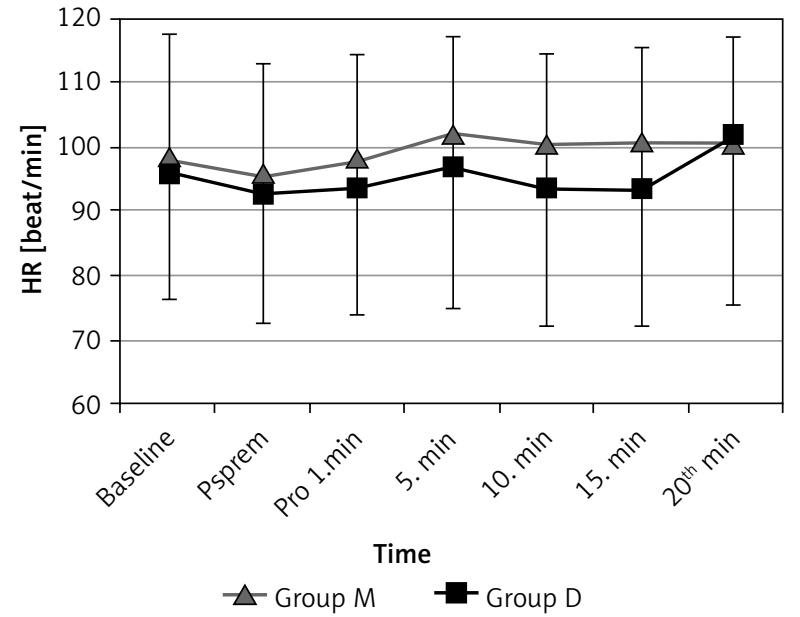

Figure 2. Changes in heart rate (HR) values in two groups

Values are expressed as mean \pm SD. Baseline (before sedation), Psprem (after premedication), Pro 1. min (1 ${ }^{\text {st }}$ min of the procedure), 5. $\min \left(5^{\text {th }} \mathrm{min}\right.$ of the procedure), 10. $\mathrm{min}\left(10^{\text {th }} \mathrm{min}\right.$ of the procedure), 15. $\min$ (15 th $\min$ of the procedure), 20. $\min \left(20^{\text {th }}\right.$ min of the procedure).

$p<0.001)$. Additionally, eye-opening, verbal response and cooperation times were significantly lower in the dexmedetomidine group, as shown in Table II. In both groups, BIS values of $70-80$, which indicate the targeted sedation level, were reached. The mean \pm SD BIS scores are shown in Table III. Total propofol consumption was compared between

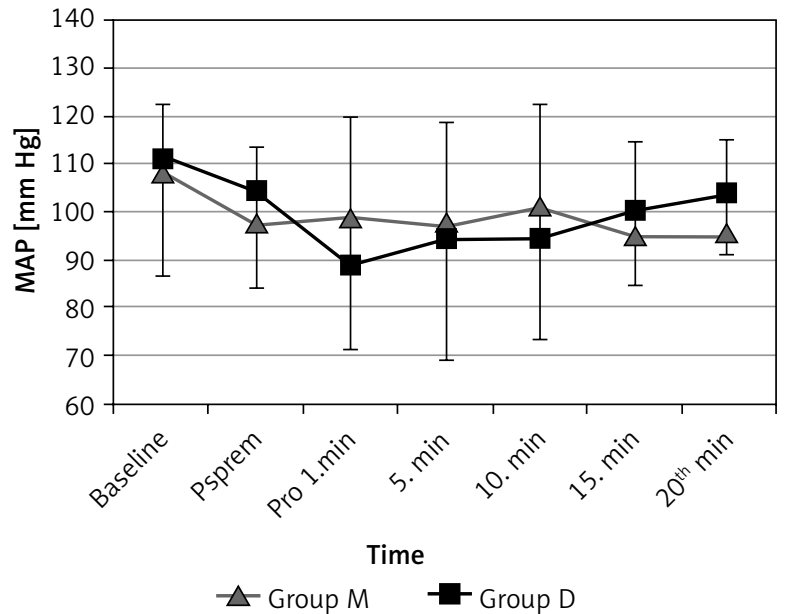

Figure 3. Changes in mean arterial blood pressure (MAP) values in two groups

Values are expressed as mean $\pm S D$. Baseline (before sedation), Psprem (after premedication), Pro 1. min (1 $1^{\text {st }}$ min of the procedure), 5. $\mathrm{min}$ ( $5^{\text {th }} \mathrm{min}$ of the procedure), 10. $\mathrm{min}$ (10 $10^{\text {th }} \mathrm{min}$ of the procedure), 15. $\min$ (15 th $\min$ of the procedure), 20. $\min \left(20^{\text {th }}\right.$ min of the procedure).

groups. In the midazolam group, cumulative propofol doses were significantly higher $(208.5 \pm 80.0 \mathrm{mg}$ vs. $154.5 \pm 66.7 \mathrm{mg} ; p<0.01)$ than in the dexmedetomidine group (Figure 4).

When hemodynamic variables were analysed with in-group analysis, we observed that heart rate values decreased after premedication and after

Table II. Recovery time variables between groups

\begin{tabular}{|lclc|}
\hline Parameter & Group M & Group D & $P$-value \\
\hline Time to reach Aldrete score 9 [min] & $9.4 \pm 2.1$ & $6.6 \pm 1.3^{*}$ & $<0.001$ \\
\hline Eye opening time [min] & $6.9 \pm 1.9$ & $4.8 \pm 1.5^{*}$ & $<0.001$ \\
\hline Verbal response [min] & $8.5 \pm 2.0$ & $6.1 \pm 1.5^{\star}$ & $<0.001$ \\
\hline Cooperation time [min] & $10.3 \pm 2.7$ & $7.7 \pm 2.0^{*}$ & $<0.001$ \\
\hline
\end{tabular}

${ }^{*} P<0.05$ is considered statistically significant.

Table III. Mean BIS values in groups

\begin{tabular}{|lccc|}
\hline Parameter & Group M & Group D & $P$-value \\
\hline Baseline & $93.3 \pm 0.64$ & $94.8 \pm 0.38$ & NS \\
\hline After premedication & $81.1 \pm 0.89$ & $82.55 \pm 0.72$ & NS \\
\hline After propofol $5^{\text {th }}$ min & $71.8 \pm 0.89$ & $70.95 \pm 0.81$ & NS \\
\hline After propofol $10^{\text {th }}$ min & $73.1 \pm 0.72$ & $74.15 \pm 0.69$ & NS \\
\hline After propofol $15^{\text {th }}$ min & $71.65 \pm 0.87$ & $70.95 \pm 0.81$ & NS \\
\hline After propofol $20^{\text {th }}$ min & $72.35 \pm 0.77$ & $73.65 \pm 0.68$ & NS \\
\hline
\end{tabular}

${ }^{*} P<0.05$ is considered statistically significant. NS - non-significant. 


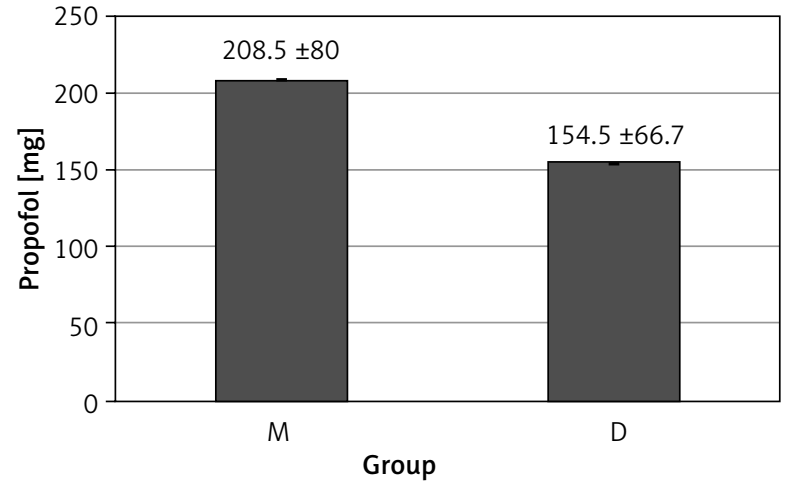

Figure 4. Cumulative dose of propofol in groups

Values are expressed as mean $\pm S D$.

propofol bolus injection in both groups. This decrease was an expected effect secondary to the sedation. However, the decrease did not exceed a level that was $20 \%$ over baseline values, as shown in Figure 2 .

There was no arrhythmia, apnoea, shivering or bradycardia (> 20\% decrease to baseline) in either group. Hypotension occurred in 1 patient in group $M$ and 3 patients in group $D(p=0.302)$. Nausea/vomiting was observed in 3 patients in group $M$ and none in group $D(p=0.231)$. Five patients in group $M$ and 4 patients in group D needed oxygen supply ( $25 \%$ vs. $20 \%$, respectively; $p>0.05$ ). Side effects were similar, as shown in Table IV.

\section{Discussion}

In this study, we found that patients receiving dexmedetomidine-propofol sedation during ERCP had a significantly shorter recovery time. Less propofol consumption in the dexmedetomidine group was thought to have affected this result. The changes in respiratory and haemodynamic variables were similar in both groups.

Adequate sedation during endoscopic procedures, especially for interventional ERCP, directly affects the operation time and success. Propofol is widely used for this purpose, and it is postulated to be effective in sedation for ERCP. In a meta-analysis, propofol-induced respiratory depression and hypotensive effects were shown to be more common than in single use. In the same article, it was reported that recovery time was shorter and patient cooperation was better when used with opioids or midazolam [11]. However, higher doses of propofol may cause platelet aggregation [12], metabolic acidosis [13], delayed awakening [14], depression of the hypoxic ventilator response, and cardiorespiratory depression. Therefore, to decrease these adverse effects of propofol, it is commonly combined with other sedatives. Peden et al. [15] reported that the addition of dexmedetomidine to propofol caused a reduction in the propofol requirement and a decrease in the plasma concentrations of propofol. In our study, we found statistically significantly lower propofol consumption in the dexmedetomidine group.

In a study, it was demonstrated that conscious sedation for diagnostic and therapeutic ERCP can be successfully and safely achieved using midazolam [16].

In a randomized, double-blind, prospective clinical trial, the authors compared propofol plus fentanyl with dexmedetomidine infusion alone. These researchers found that patients needed more additional analgesics in the dexmedetomidine group. The authors also concluded that dexmedetomidine was associated with higher haemodynamic instabil-

Table IV. Adverse effects in groups

\begin{tabular}{|lccc|}
\hline Parameter & Group M $(n=20)$ & Group D $(n=20)$ & $P$-value \\
\hline$>20 \mathrm{~mm}$ Hg decreases in MAP & $1 / 20(5 \%)$ & $3 / 20(15 \%)$ & NS \\
\hline$>20 \mathrm{~mm}$ Hg increases in MAP & $0 / 20(0 \%)$ & $1 / 20(5 \%)$ & NS \\
\hline$>20 \%$ increase in HR & $0 / 20(0 \%)$ & $1 / 20(5 \%)$ & NS \\
\hline$>20 \%$ decrease in HR & $0 / 20(0 \%)$ & $0 / 20(0 \%)$ & NS \\
\hline Nausea-vomiting & $3 / 20(15 \%)$ & $0 / 20(0 \%)$ & NS \\
\hline Shivering & $0 / 20(0 \%)$ & $0 / 20(0 \%)$ & NS \\
\hline Apnoea & $0 / 20(0 \%)$ & $0 / 20(0 \%)$ & NS \\
\hline Arrhythmia & $0 / 20(0 \%)$ & $0 / 20(0 \%)$ & NS \\
\hline Supplemental oxygen & $5 / 20(25 \%)$ & $4 / 20(20 \%)$ & \\
\hline
\end{tabular}

$P<0.05$ considered statistically significant. NS - non-significant. 
ity and prolonged recovery [17]. This finding is different from the findings of our study. We think that this difference may be due to high doses of dexmedetomidine, which was used alone during the entire procedure in that study. In our study, we only applied dexmedetomidine or midazolam before the procedure. Sedation maintenance was supplied with propofol. The recovery time was shorter in our study because we used dexmedetomidine in combination with a lower cumulative dose of propofol. Both the lower doses of dexmedetomidine and propofol may be effective for shorter recovery times. Seifert et al. [3] compared propofol alone and propofol-midazolam combinations in interventional endoscopy. The authors found similar sedative efficacy in both groups but longer recovery times in the propofol-midazolam combination group (19 \pm 7 vs. $25 \pm 9 \mathrm{~min}$; $p<0.01)$. This finding may be due to the relatively slower elimination half-life of midazolam. In our study, the shorter recovery time in the dexmedetomidine group may be secondary to both the short elimination half-life of dexmedetomidine and less propofol consumption.

A study by Lee et al. [18] compared the sedative effect and adverse events of midazolam-meperidine-dexmedetomidine and midazolam-meperidine during ERCP and found that adding dexmedetomidine to the midazolam-meperidine regimen was more effective and safe during ERCP compared with a midazolam-meperidine regimen.

We used BIS measurements to objectively achieve adequate sedation levels. Thus, the total sedative agent dose did not depend on the operator's subjective evaluation. We found that the total propofol consumption was lower in group D (154.5 \pm 66.7 vs. $208.5 \pm 80.0 \mathrm{mg} ; p=0.011$ ). This result is important, and it may be postulated that dexmedetomidine might be a good alternative for sedation with the propofol sparing effect. It can be postulated that due to less propofol consumption in the dexmedetomidine group, cost-effectiveness in this group is better. This approach reduces the possible respiratory depressive effect of propofol by decreasing the total consumption. In an experimental study with a rabbit model, the researchers found that ventilator depression was higher in treatment with propofol and midazolam. The depression was lowered with dexmedetomidine [19]. This outcome is an important respiratory protective effect for ambulatory sedation.
Dexmedetomidine has been associated with decreases in HR because of its $\alpha 2$ agonism and sympatholytic effect [20]. In a study, significant decreases in MAP and HR occurred if dexmedetomidine was used as monotherapy $[17,20]$. This effect may be due to the higher doses of the drug, and decreased sympathetic outflow and circulating catecholamine levels [21]. Gastrointestinal endoscopy studies using dexmedetomidine and midazolam showed that the two agents do not differ from each other in terms of hypoxia, bradycardia and hypotensive effects [22]. Again, in this meta-analysis, patients who were treated with dexmedetomidine for longer procedures, such as ERCP or endoscopic mucosal resection, reported less restlessness [23]. In our study, we did not find any respiratory or hemodynamic differences (MAP and HR) between the groups. In group analysis, the MAP decreased during the procedure but did not exceed $20 \%$ compared to the baseline values. The most important cause of hemodynamic side effects due to dexmedetomidine is the high-speed and longterm induction dose [24]. It has also been reported that it may have hypertensive effects when used as the sole agent [25]. In our study, dexmedetomidine was used in induction together with propofol at one time and $1 \mu \mathrm{g} / \mathrm{kg}$ (10 min). Infusion treatment was not given. A hemodynamic side effect was not clearly observed due to the reasons mentioned above.

Our study has limitations. Recovery times between the two groups are very similar. The reason for this is that we have worked with a very experienced (> 3000 cases) gastroenterologist because the duration of the procedure in our study is relatively short compared to other studies [22].

In a study in which propofol was used in combination with dexmedetomidine and midazolam, side effects such as restlessness and agitation were observed as a side effects Purshka. These effects are insufficient sedation findings. In this study, sedation may not be evaluated objectively because sedation was performed according to the Ramsey sedation score. When the side effects were examined in our study, no difference was observed in terms of restlessness between the groups, since sedation was achieved at the same level as BIS monitorization.

There was no difference in nausea-vomiting between the groups because antiemetic treatment was applied in both groups. 


\section{Conclusions}

In this randomized, double-blind, prospective study, we found that the dexmedetomidine-propofol combination had a shorter recovery time and similar sedative and adverse effects compared with the midazolam-propofol combination. On the other hand, our study suggests that adjunctive use of dexmedetomidine reduces propofol requirements during ERCP. Therefore, we can conclude that the combination of dexmedetomidine and propofol may be a safe and useful alternative for sedation and dose reduction in ERCP patients.

\section{Conflict of interest}

The authors declare no conflict of interest.

\section{References}

1. Li S, Sheng G, Teng Y, Sun M. Systematic review of anaesthet ic medication for ERCP based on a network meta-analysis. Int J Surg 2018; 51: 56-62.

2. Claeys MA, Gepts E, Camu F. Haemodynamic changes during anaesthesia induced and maintained with propofol. $\mathrm{Br} J \mathrm{An}$ aesth 1988; 60: 3-9.

3. Seifert H, Schmitt TH, Gültekin T, et al. Sedation with propofol plus midazolam versus propofol alone for interventional endoscopic procedures: a prospective, randomized study. Aliment Pharmacol Ther 2000; 14: 1207-14.

4. Kogan A, Efrat R, Katz J, Vidne BA. Propofol-ketamine mixture for anesthesia in pediatric patients undergoing cardiac catheterization. J Cardiothorac Vasc Anesth 2003; 17: 691-3.

5. Le Guen M, Liu N, Tounou F, et al. Dexmedetomidine reduces propofol and remifentanil requirements during bispectral index-guided closed-loop anesthesia: a double-blind, placebocontrolled trial. Anesth Analg 2014; 118: 946-55.

6. Bower AL, Ripepi A, Dilger J, et al. Bispectral index monitoring of sedation during endoscopy. Gastrointest Endosc 2000; 52: 192-6.

7. Ramkiran S, Iyer SS, Dharmavaram S, et al. BIS targeted propofol sparing effects of dexmedetomidine versus ketamine in outpatient ERCP: a prospective randomised controlled trial. J Clin Diagn Res 2015; 9: UC07-12.

8. Kearse LA, Rosow C, Zaslavsky A, et al. Bispectral analysis of the electroencephalogram predicts conscious processing of information during propofol sedation and hypnosis. Anesthesiology 1998; 88: 25-34.

9. Jokelainen J, Mustonen H, Kylänpää L, et al. Assessment of sedation level for endoscopic retrograde cholangiopancreatography - a prospective validation study. Scand J Gastroenterol 2018; 53: 370-5.

10. Aldrete JA, Kronlik D. A postanesthetic recovery score. Anest Analg 1970; 49: 924-34.

11. Zhang R, Lu Q, Wu Y. The comparison of midazolam and propofol in gastrointestinal endoscopy: a systematic review and meta-analysis. Surg Laparosc Endosc Percutan Tech 2018; 28: 153-8.

12. Aoki H, Mizobe T, Nozuchi S, Hiramatsu N. In vivo and in vitro studies of the inhibitory effect of propofol on human platelet aggregation. Anesthesiology 1998; 88: 362-70.

13. Cravens GT, Packer DL, Johnson ME. Incidence of propofol infusion syndrome during noninvasive radiofrequency ablation for atrial flutter or fibrillation. Anesthesiology 2007; 106: 1134-8.

14. Pascoe PJ, Ilkiw JE, Frischmeyer KJ. The effect of the duration of propofol administration on recovery from anesthesia in cats. Vet Anaesth Analg 2006; 33: 2-7.

15. Peden CJ, Cloote AH, Stratford N, Prys-Roberts C. The effect of intravenous dexmedetomidine premedication on the dose requirement of propofol to induce loss of consciousness in patients receiving alfentanil. Anaesthesia 2001; 56: 408-13.

16. Yuksel O, Parlak E, Koklu S, et al. Conscious sedation during endoscopic retrograde cholangiopancreatography: midazolam or midazolam plus meperidine? Eur J Gastroenterol Hepatol 2007; 19: 1002-6.

17. Muller S, Borowics SM, Fortis EA, et al. Clinical efficacy of dexmedetomidine alone is less than propofol for conscious sedation during ERCP. Gastrointest Endosc 2008; 67: 651-9.

18. Lee BS, Ryu J, Lee SH, et al. Midazolam with meperidine and dexmedetomidine vs. midazolam with meperidine for sedation during ERCP: prospective, randomized, double-blinded trial. Endoscopy 2014; 46: 291-8.

19. Chang C, Uchiyama A, Ma L, et al. Comparison of the effects on respiratory carbon dioxide response, arterial blood pressure, and heart rate of dexmedetomidine, propofol, and midazolam in sevoflurane-anesthetized rabbits. Anesth Analg 2009; 109: 84-9.

20. Erdman MJ, Doepker BA, Gerlach AT, et al. A comparison of severe hemodynamic disturbances between dexmedetomidine and propofol for sedation in neurocritical care patients. Crit Care Med 2014; 42: 1696-702.

21. Naaz S, Ozair E. Dexmedetomidine in current anaesthesia practice - a review. J Clin Diagn Res 2014; 8: GE01-4.

22. Pushkarna G, Sarangal P, Pushkarna V, Gupta R. Comparative evaluation of dexmedetomidine versus midazolam as premedication to propofol anesthesia in endoscopic retrograde cholangiopancreatography. Anesth Essays Res 2019; 13: 297-302.

23. Nishizawa T, Suzuki H, Sagara S, et al. Dexmedetomidine versus midazolam for gastrointestinal endoscopy: a meta-analysis. Dig Endosc 2015; 27: 8-15.

24. Jakob SM, Ruokonen E, Grounds RM, et al. Dexmedetomidine for long-term sedation I: Dexmedetomidine vs midazolam or propofol for sedation during prolonged mechanical ventilation: two randomized controlled trials. JAMA 2012; 307: 1151-60.

25. Kontak AC, Victor RG, Vongpatanasin W. Dexmedetomidine as a novel countermeasure for cocaine-induced central sympathoexcitation in cocaine-addicted humans. Hypertension 2013; 61: 388-94.

Received: 1.03.2020, accepted: 14.04.2020. 